\title{
A NOTE ON THE PLANE JACOBIAN CONJECTURE
}

\author{
NGUYEN VAN CHAU
}

\begin{abstract}
It is shown that every polynomial function $P: \mathbb{C}^{2} \longrightarrow \mathbb{C}$ with irreducible fibres of same a genus is a coordinate. In consequence, there does not exist counterexamples $F=(P, Q)$ to the Jacobian conjecture such that all fibres of $P$ are irreducible curves of same a genus.

Keywords and Phrases: Jacobian conjecture, Rational curve. 2000 Mathematical Subject Classification: 14R15, 14E22, 14D06,14H05.
\end{abstract}

1. The Jacobian conjecture (JC), posed first in 1939 by Ott-Heinrich Keller 7 ] and still opened, asserts that every polynomial maps $F=(P, Q): \mathbb{C}^{2} \longrightarrow \mathbb{C}^{2}$ with non-zero constant Jacobian $J(P, Q):=P_{x} Q_{y}-P_{y} Q_{x} \equiv c \neq 0$ is invertible. Its history, many references, and some partial results, can be found in [2] and [4.

Since 1979 in his work 15 concerning with the plane case of (JC) Razar had found the following:

Theorem 1 ([15]). A non-zero constant Jacobian polynomial map $F=(P, Q)$ is invertible if all fibres of $P$ are irreducible rational curves.

In other words, it claim that there does not exists counterexamples $F=(P, Q)$ to (JC) such that all fibres of $P$ are irreducible rational curves. In attempting to understand the nature of the plane Jacobian conjecture, Razar 's result had been reproved by Heitmann [6], Lê and Weber [10, Friedland [14, Nemethi and Sigray 12 in several different algebraic and algebro-geometric approaches. It was also proved in 12 that there does note exists counterexamples $F=(P, Q)$ to (JC) such that all fibres of $P$ are irreducible elliptic curves. Following [13, a polynomial function $f: \mathbb{C}^{2} \longrightarrow \mathbb{C}$ is called coordinate if there is $g \in \mathbb{C}[x, y]$ such that $(f, g): \mathbb{C}^{2} \longrightarrow \mathbb{C}^{2}$ is a polynomial automorphism. In fact, Vistoli 17 and Neumann and Norbury [13] obtained the following, which implies Theorem[1]

Theorem 2. A polynomial function $P: \mathbb{C}^{2} \longrightarrow \mathbb{C}$ with irreducible rational fibres is a coordinate.

This theorem, as mentioned in 13, is implicit in Myianishi's earlier work 11 (Lemma 1.7). In this article we would like to present the following improvement of Theorem 2

Theorem 3. A polynomial function $P: \mathbb{C}^{2} \longrightarrow \mathbb{C}$ with irreducible fibres of same a genus is a coordinate.

Here, as usual, we mean the genus of an irreducible algebraic curve to be the genus of the desingularization of this curve. In other words, Theorem 3 says that the genus of the fibres of any polynomial with irreducible fibres must vary, except for coordinates. In view of this theorem there does not exist counterexamples

This research is supposed by NAFOSTED, Vietnam. 
$F=(P, Q)$ to $(\mathrm{JC})$ such that all fibres of $P$ are irreducible curves of same a genus. Note that, following Kaliman [8, to prove the plane case of $(\mathrm{JC})$ it is sufficient to consider only non-zero constant Jacobian polynomial maps $F=(P, Q)$ in which all fibres of $P$ are irreducible. Further investigations on how the genus of the fibres of polynomial functions in $\mathbb{C}^{2}$ change should be useful in hunting the solution of the plane Jacobian conjecture.

The proof of Theorem 3, presented in Section 2, is a combination of Theorem 2 and the fact that if the fibres of $P$ are irreducible curves of same a genus $g$, then $g=0$ (Lemma 1 and Lemma 2). The proofs of Theorem 2 in [13, [11] and [17. use Abhyankar-Moh-Suzuki Embedding Theorem, or Zariski's main theorem. In Section 3 we will give another elementary proof of Theorem 2 by applying NewtonPuiseux Theorem and the basic properties of the standard resolution of singularities.

2. Let $P \in \mathbb{C}[x, y]$ be a given non-constant polynomial. Regard the plane $\mathbb{C}^{2}$ as a subset of the projective plane $\mathbb{P}^{2}$ and consider $P$ as a rational morphism $P: \mathbb{P}^{2} \longrightarrow$ $\mathbb{P}$, which is well defined everywhere on $\mathbb{P}^{2}$, except a finite number of points in the line at infinity $z=0$. By blowing-up we can remove indeterminacy points of $P$ and obtain a compactification $X$ of $\mathbb{C}^{2}$ and a regular extension $p: X \longrightarrow \mathbb{P}$ of $P$ over $X$. The divisor $\mathcal{D}:=X \backslash \mathbb{C}^{2}$ is the union of smooth rational curves with simple normal crossings. An irreducible component $D$ of $\mathcal{D}$ is called horizontal curve of $P$ if $p_{\mid D}$ is a non-constant mapping. Note that the number of horizontal curves of $P$ does not depend on the regular extension $p$. Following [9] such a compactification $X$ is called minimal if among components of $\mathcal{D}$ the only the proper transform of the line at infinity $z=0$ and horizontal curves may have self-intersections -1 . A regular extension of $P$ over a minimal compactification of $\mathbb{C}^{2}$ can be constructed by a blowing-up process in which we blow up only at the indeterminacy points of $P$ and of the resulted blowing-up versions of $P$.

Now, suppose $p: X \longrightarrow \mathbb{P}^{1}$ is a regular extension of $P$ over a minimal compactification $X$ of $\mathbb{C}^{2}$. Let us denote by $C_{s}$ the fiber of $p$ over $s \in \mathbb{P}^{1}$ and by $C$ the generic fiber of $p$. Let $\mathcal{D}_{s}:=\mathcal{D} \cap C_{s}$ - the portion of $C_{s}$ contained in $\mathcal{D}$. The minimality of the compactification $X$ of $\mathbb{C}^{2}$ yields the following usefulness:

$\left(^{*}\right)$ Every irreducible components in $\mathcal{D}_{s}, s \in \mathbb{C}$, must has self-intersections less than -1 .

We begin with the following observation.

Lemma 1. Assume that all fibres of $P$ are irreducible curves of same a genus $g$. Then, the fibres $C_{s}, s \in \mathbb{C}$, are smooth irreducible curves of same genus $g$.

Proof. As usual, we denote by $K_{X}$ the canonical bundle of the surface $X$, by $\pi(V)$ the virtual genus of an algebraic curve $V$ in $X$, and by $g(V)$ the genus of the desingularization of $V$ for when $V$ is irreducible. By the adjunction formula

$$
2 \pi(V)-2=K_{X} \cdot V+V \cdot V,
$$

and, for irreducible curve $V, \pi(V)=g(V)$ if and only if $V$ is smooth. Furthermore, if $V$ is a fiber of a fibration over $X$, then $V \cdot V=0$ and hence

$$
2 \pi(V)-2=K_{X} \cdot V,
$$

(see, for example, in [5]). For $s \in \mathbb{C}$ let us denote by $F_{s}$ the closure in $X$ of the curve $\left\{(x, y) \in \mathbb{C}^{2}: P(x, y)=s\right\}$. By assumptions the curves $F_{s}$ are irreducible 
and

$$
g\left(F_{s}\right) \equiv g, s \in \mathbb{C} .
$$

Now, let $s \in \mathbb{C}$ be given. By the adjunction formula (see in [5])

$$
2 g-2=K_{X} \cdot C_{s} .
$$

If $C_{s}$ is irreducible, we have $C_{s}=F_{s}$ and $F_{s} \cdot F_{s}=0$. Again by the adjunction formula we get $2 \pi\left(F_{s}\right)-2=K_{X} \cdot F_{s}$. Therefore, by (1) and (2) we obtain $\pi\left(F_{s}\right)=$ $g=g\left(F_{s}\right)$. Thus, $C_{s}$ is a smooth irreducible curves of genus $g$. So, to complete the proof we need to show only that $C_{s}$ is irreducible. Indeed, assume the contrary that $C_{s}$ is not irreducible. Write

$$
C_{s}=\sum_{i=1}^{k} m_{i} C_{i}+n F_{s}
$$

where $C_{i}$ are irreducible components of $\mathcal{D}_{s}$ with multiplicity $m_{i}$ and $n$ is the multiplicity of $F_{s}$ in $C_{s}$. The equality (2) becomes

$$
2 g-2=\sum_{i=1}^{k} m_{i} K_{X} \cdot C_{i}+n K_{X} \cdot F_{s} .
$$

Since $C_{i}$ are smooth irreducible rational curves, $\pi\left(C_{i}\right)=0$. Furthermore, $C_{i} . C_{i}<$ -1 by Property $(*)$ and $F_{s}^{2}<0$ by Zariski's lemma (see, for example, [1]). Then, applying the adjunction formula to $C_{i}$ and $F_{s}$ we have

$$
K_{X} \cdot C_{i}=-\left(C_{i} \cdot C_{i}+2\right) \geq 0
$$

and

$$
K_{X} \cdot F_{s}=2 \pi\left(F_{s}\right)-2-F_{s} \cdot F_{s}>2 \pi\left(F_{s}\right)-2 .
$$

From the above estimations and (3) it follows that $2 g-2>2 \pi\left(F_{s}\right)-2$. This is impossible, since $\pi\left(F_{s}\right) \geq g\left(F_{s}\right)=g$. Hence, $C_{s}$ must be irreducible.

Lemma 2. Let $P$ be as in Lemma 1. Then, $g=0$ and $P$ has only one horizontal curve.

Proof. We will use Suzukis formula [16

$$
\sum_{s \in \mathbb{P}^{1}} \chi\left(C_{s}\right)-\chi(C)=\chi(X)-2 \chi(C)
$$

Here, $\chi(V)$ indicates the Euler-Poincare characteristic of $V$. Let us denote by $m$ the number of irreducible components of the divisor $\mathcal{D}$, by $m_{\infty}$ the number of irreducible components of $C_{\infty}$ and by $h$ the number of the horizontal curves of $P$. Note that $\chi(X)=2+m$ and $\chi\left(C_{\infty}\right)=1+m_{\infty}$. Furthermore, in view of Lemma 1 1 $\chi\left(C_{s}\right)=\chi(C)=2-2 g$ for all $s \in \mathbb{C}$ and $h=m-m_{\infty}$. Now, by the above estimations we have

$$
\sum_{s \in \mathbb{P}^{1}} \chi\left(C_{s}\right)-\chi(C)=\chi\left(C_{\infty}\right)-\chi(C)=1+m_{\infty}-(2-2 g)
$$

and

$$
\chi(X)-2 \chi(C)=2+m-2(2-2 g) .
$$

Then, the equality (4) becomes

$$
1+m_{\infty}-(2-2 g)=2+m-2(2-2 g),
$$


or equivalent,

$$
2 g=1-\left(m-m_{\infty}\right)=1-h .
$$

Since $g \geq 0$ and $h \geq 1$, it follows that $g=0$ and $h=1$.

Proof of Theorem 3. Combining Lemma 2 with Theorem 2 ,

3. The main arguments in the proofs of Theorem 22 in [13, [17] and [1], leads to the fact that if the fibres of $P$ are rational irreducible curves, then $P$ has only one horizontal curve and its fibres are isomorphic to $\mathbb{C}$. The fact that $P$ is a coordinate then follows from Abhyankar-Moh-Suzuki Embedding Theorem, as done in 11] (Lemma 1.7), or from Zariski's main theorem, as in [17.(Lemma 4.8). Instead of such ways, Theorem 2 can also be proved by using the observations below.

i) Let $H \in \mathbb{C}[x, y], H(x, y)=\sum_{i j} c_{i j} x^{i} y^{j}$. Recall that the so-called Newton polygon $N_{H}$ of $H$ is the convex hull of the set $\{(0,0)\} \cup\left\{(i, j): c_{i j} \neq 0\right\}$.

Fact 1. Assume that $\operatorname{deg}_{x} H>0$ and $\operatorname{deg}_{y} H>0$. If $H$ has only one horizontal curve, then $N_{H}$ is a triangle with vertices $(0,0),\left(\operatorname{deg}_{x} H, 0\right)$ and $\left(0, \operatorname{deg}_{y} H\right)$ and the summation $H_{E}$ of monomials $c_{i j} x^{i} y^{j}$ in $H$ with $(i, j)$ lying in the edge joining $\left(\operatorname{deg}_{x} H, 0\right)$ and $\left(0, \operatorname{deg}_{y} H\right)$ is of the form

$$
H_{E}(x, y)=C\left(y^{q}-a x^{p}\right)^{m}
$$

where $C \neq 0, a \neq 0, p, q, m$ are natural numbers and $\operatorname{gcd}(p, q)=1$.

In fact, if $H$ has only one horizontal curve, the branches at infinity of any generic fiber $H=c$ can be given by Newton-Puiseux expansions of same one of the forms

$$
y=b x^{p / q}+\text { lower terms in } x, p / q \leq 1
$$

and

$$
x=b y^{q / p}+\text { lower terms in } y, q / p \leq 1,
$$

where $b \neq 0$ and $\operatorname{gcd}(p, q)=1$. The Newton polygon $N_{H}$ and the face polynomial $H_{E}$ then can be detected by using Newton-Puiseux theorem and the basic properties of Newton-Puiseux expansions (see [3]).

ii) Let $P \in \mathbb{C}[x, y]$ with $\operatorname{deg} P>1, \operatorname{deg}_{x} P>0$ and $\operatorname{deg}_{y} P>0$. Assume that the fibres of $P$ are irreducible rational curves, and hence, $P$ has only one horizontal curve. In view of Fact 1 , we can assume that $P_{E}(x, y)=C\left(y^{q}-a x^{p}\right)^{m}$ with $C \neq 0$, $a \neq 0$ and $\operatorname{gcd}(p, q)=1$.

Fact 2. $P_{E}(x, y)$ is of the forms $C\left(y^{q}-a x\right)^{m}$ and $C\left(y^{q}-a x\right)^{m}, q \geq 1$.

To see it, we need consider only the case $p / q \neq 1$. Assume, for example, that $p / q<1$. Let $p: X \longrightarrow \mathbb{P}^{1}$ be a regular extension over a minimal compactification $X$ of $\mathbb{C}^{2}$, which results from a blowing-up process $\pi: X \longrightarrow \mathbb{P}^{2}$ that blow up only at the indeterminacy points of $P$ and of the obtained blowing-up versions of $P$. By Lemma 1 the divisor at infinity $\mathcal{D}$ is the union of the fiber $C_{\infty}$ and the unique horizontal curve $D, \mathcal{D}=C_{\infty} \cup D$. The fiber $C_{\infty}$ contains the proper transform $D_{0}$ of the line at infinity $z=0$ of $\mathbb{C}^{2}$. Since $\operatorname{deg} P>1$ and the morphism $p: X \longrightarrow \mathbb{P}^{1}$ is a $\mathbb{P}^{1}$-fibration, the fiber $C_{\infty}$ is reducible and can be contracted to one smooth rational curve by blowing down. Furthermore, $D_{0}$ is the unique component of $C_{\infty}$ 
having self-intersection -1 . Then, one can see that the first Puiseux chain of the dual graph of $\mathcal{D}$ must be of the form

$$
\stackrel{D_{0}}{\bullet_{-1}}-\underset{-2}{\stackrel{e_{1}}{0}}-\cdots-\underset{-2}{\stackrel{e_{k}}{\circ}}-\underset{-2}{\bigcirc}-f_{0}^{f_{1}}-\cdots-\stackrel{f_{l}}{\circ},
$$

where the weights are the self-intersection numbers. This Puiseux chain coincides with the resolution graph of the germ curve at infinity $\gamma$, composed of the line $z=0$ and a branch curve at infinity given by a Newton-Puiseux expansion of the form

$$
y=b x^{p / q}+\text { lower terms in } x, b \neq 0 .
$$

Note that the line at infinity $z=0$ of $\mathbb{C}^{2}$ has self-intersection 1 . Then, by examining in detail the resolving singularities of $\gamma$ we can easily see that the self-intersection conditions $D_{0}^{2}=-1$ and $e_{i}^{2}=-2, i=1, k$, are satisfied only when $p=1, q>1$ and $l=1$.

Thus, once the fibres of $P$ are irreducible rational curves, Fact 2 enables us to easily construct polynomial automorphisms $\Phi$ of $\mathbb{C}^{2}$ such that $\operatorname{deg} P \circ \Phi(x, y)=1$.

4. Let us to conclude here by a remark that a non-zero constant Jacobian polynomial map $F=(P, Q)$ is invertible if $P$ has only one horizontal curve. This is a key point of the geometric proof of Theorem 1 presented by Lê and Weber in 10. In fact, if $P$ has only one horizontal curve, by the Jacobian condition in regular extensions of $P$ the restriction of $Q$, viewed as a rational map, to the unique horizontal curve of $P$ must be a constant mapping with value $\infty$. So, the restriction of $Q$ to a generic fiber $P=c$ is proper. Therefore, by the simply connectedness of $\mathbb{C}$ such a generic fiber $P=c$ is isomorphic to $\mathbb{C}$. Hence, $(P, Q)$ is invertible.

It seems to be very difficult to estimate the number of horizontal curves of polynomial components of non-zero constant Jacobian polynomial maps of $\mathbb{C}^{2}$.

Acknowledgements. The author would like to express his thank to Pierete Cassou-Nogues, Mutsui Oka and Ha Huy Vui for many valuable discussions and helps.

\section{REFERENCES}

[1] W. Barth, C. Peters, A. van de Ven, Compact complex surfaces, Ergebnisse der Mathematik und ihrer Grenzgebiete (3), 4 (Springer-Verlag, Berlin-New York, 1984).

[2] H. Bass, E. Connell and D. Wright, The Jacobian conjecture: reduction of degree and formal expansion of the inverse, Bull. Amer. Math. Soc. (N.S.) 7 (1982), 287-330.

[3] E. Brieskorn and H. Knorrer, Ebene algebraische Kurven, Birkhauser, Basel-BostonStuttgart, 1981.

[4] Van den Essen, Arno, Polynomial automorphisms and the Jacobian conjecture, Progress in Mathematics 190, Birkhauser, Basel, 2000.

[5] P. Griffiths and J. Harris, Principles of algebraic geometry, John Wiley, New York, 1978.

[6] R. Heitmann, On the Jacobian conjecture, J. Pure Appl. Algebra 64 (1990), 36-72 and Corrigendum ibid. 90 (1993), 199-200.

[7] Ott-Heinrich Keller, Ganze Cremona-Transformatione, Monatsh. Math. Phys. 47(1939), 299306.

[8] Sh. Kaliman, On the Jacobian conjecture, Proc. Amer. Math. Soc., Vol. 117, No. 1, January 1993, 45-51.

[9] Lê Dũng Tráng, Simple rational polynomials and the Jacobian conjecture, Publ. RIMS, Kyoto Univ. 44 (2008), 641-659.

[10] Lê Dũng Tráng and C. Weber, Polynômes à fibres rationnelles et conjecture jacobienne 2 variables, C. R. Acad. Sci. Paris Sr. I Math. 320 (1995), 581-584. 
[11] M. Miyanishi and T. Sugie, Generically rational polynomials, Osaka J. Math. 17 (1980), 339362.

[12] Némethi, A. and Sigray, I. On the monodromy representation of polynomial maps in $n$ variables. Studia Sci. Math. Hungar. 39 (2002), no. 3-4, 361-367.

[13] W.D. Neumann and P. Norbury, Nontrivial rational polynomials in two variables have reducible fibres, Bull. Austral. Math. Soc. 58 (1998), 501-503.

[14] Friedland, Shmuel. Monodromy, differential equations and the Jacobian conjecture. Ann. Polon. Math. 72 (1999), no. 3, 219-249.

[15] M. Razar, Polynomial maps with constant Jacobian. Israel J. Math. 32 (1979), 97-106.

[16] M. Suzuki, Properiété topologiques des polynômes de deux variables complexes et automorphismes algébriques de l'esppace $\mathbb{C}^{2}$, J. Math. Soc. Japan 26 (1974), 241-257.

[17] A. Vistoli, The number of reducible hypersurfaces in a pencil, Invent. Math. 112 (1993), $247-262$.

Institute of Mathematics, 18 Hoang Quoc Viet, 10307 Hanoi,Vietnam

E-mail address: nvchau@math.ac.vn 